\title{
Proposal for management and dermoscopy follow-up of nevi in patients affected by oculocutaneous albinism type la
}

\author{
Rosario Peralta ${ }^{1}$, Emilia Cohen Sabban ${ }^{2}$, Paula Friedman ${ }^{1}$, Carolina Marcucci ${ }^{1}$, \\ Luis A. Bollea Garlatti ${ }^{1}$, Gastón Galimberti ${ }^{3}$, Horacio Cabo ${ }^{2}$
}

1 Dermatology, Buenos Aires, Argentina

2 Dermatology Department, School of Medicine, Instituto de Investigaciones Médicas “A. Lanari”, University of Buenos Aires, Argentina 3 Dermatology Deparment, School of Medicine, Hospital Italiano, Buenos Aires, Argentina

Key words: dermoscopy, digital follow-up, reflectance confocal microscopy, nevi, oculocutaneous albinism, melanoma

Citation: Peralta R, Cohen Sabban E, Friedman P, Marcucci C, Bollea Garlatti LA, Galimberti G, Cabo H. Proposal for management and dermoscopy follow-up of nevi in patients affected by oculocutaneous albinism type Ia. Dermatol Pract Concept. 2017;7(1):7. DOI: https:// doi.org/10.5826/dpc.0701a07

Received: October 2, 2016; Accepted: October 3, 2016; Published: January 31, 2017

Copyright: $@ 2017$ Peralta et al. This is an open-access article distributed under the terms of the Creative Commons Attribution License, which permits unrestricted use, distribution, and reproduction in any medium, provided the original author and source are credited.

Funding: None.

Competing interests: The authors have no conflicts of interest to disclose.

All authors have contributed significantly to this publication.

Corresponding author: Rosario Peralta, MD, 12 Prefecto Furnillo St, Puerto Madryn, Chubut, Argentina. Tel. 0542804190759. Email: rosarioperalta@yahoo.com

ABSTRACT Albino patients are at great risk for developing cutaneous neoplasms, including melanomas. In this paper we describe the dermatoscopic findings of nevi in two patients with oculocutaneous albinism type Ia (OCA-Ia) highlighting that they manifest a vascular pattern similar to that described for amelanotic melanoma. We propose managing these patients with dermoscopy, using the comparative approach, digital follow up (DFU), and reflectance confocal microscopy as a complementary tool for difficult cases.

\section{Case 1}

A 55-year-old female patient with clinical diagnosis of OCAIa and a personal history of two thin amelanotic melanomas (Breslow thickness $<1 \mathrm{~mm}$ ) presented with multiple nonpigmented nevi. Clinically, these lesions were pink macules and papules. On dermoscopy, all the lesions exhibited a light pink background with regularly distributed dotted and comma-like vessels (Figure 1). The predominant vascular pattern in macular lesions was dotted vessels. In contrast, papular lesions showed predominantly comma-like vessels. None of the lesions were different from the signature pattern, and all of them were stable on time.

\section{Case 2}

A 38-year-old male patient with clinical diagnosis of OCAIa presented multiple pinkish nevi. Dermatoscopically, some nevi exhibited a pink background with regularly distributed comma-like and dotted vessels, (Figure 2 and 3 ) and others presented a homogeneous light brown, yellowish pattern associated with predominantly comma-like vessels (Figure 4). We did not identify any lesion without the mentioned signature patterns. The digital videodermoscopy follow-up showed no changes on these lesions. 


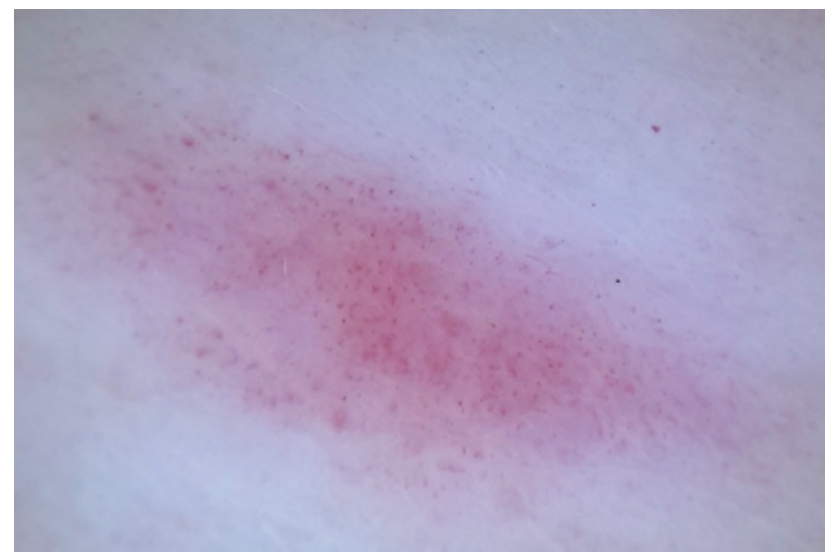

Figure 1. Nevus on back. Homogeneous light-pink background with regularly distributed dotted vessels and scarce comma-like vessels. Clinically it was a flat lesion. [Copyright: @2017 Peralta et al.]

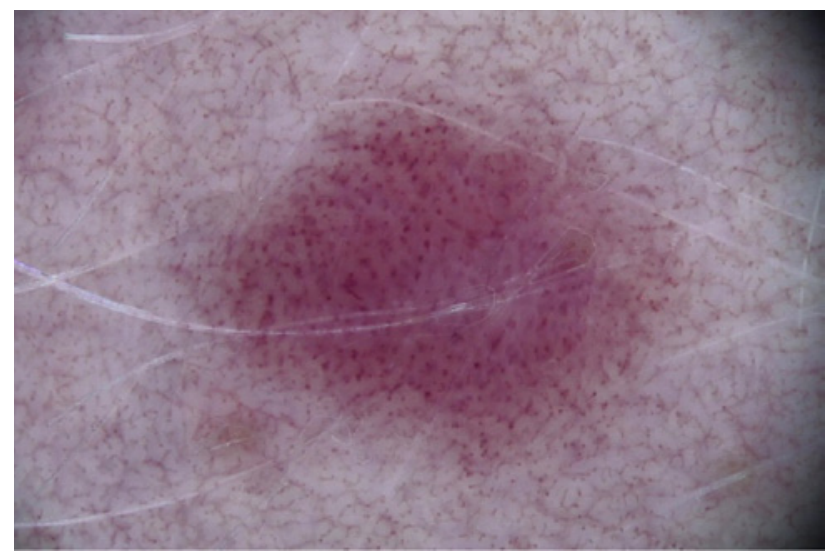

Figure 2. Nevus on left leg. Pink background with regularly distributed dotted vessels and scarce comma-like vessels. Clinically it was a macular lesion. [Copyright: (C2017 Peralta et al.]

\section{Discussion}

Oculocutaneous albinism (OCA) is a group of rare autosomal recessive disorders of pigmentation. It consists of absence or reduction of melanin in the skin, hair, and eyes due to a partial or total deficit in the activity of tyrosinase, with a normal melanocyte number and structure. The clinical spectrum of OCA varies, with OCA-Ia being the most severe type, characterized by a complete lack of melanin production, while the milder forms OCA-Ib, OCA-II, OCA-III and OCA-IV show some pigment accumulation over time. Clinically, it is possible to distinguish the most severe form (OCA-Ia), but as to the others, it is necessary to obtain a molecular diagnosis [1-4]. Clinically our patients were clearly OCA-Ia type.

To date and to our knowledge, only two reports of dermoscopic nevi features of this disease have been published in the literature [2-3].

Caldarola et al. [2] found a dermoscopic pattern represented by a homogeneous light brown, yellowish pattern associated with comma-like and dotted vessels predominantly

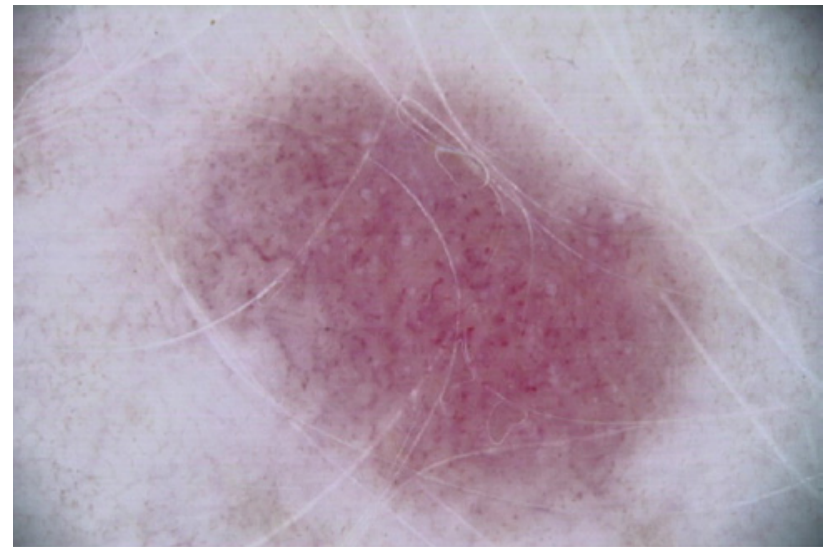

Figure 3. Nevus on right lower limb. Light pink background with regularly distributed comma-like and dotted vessels. Clinically it was a papular lesion. [Copyright: @2017 Peralta et al.]

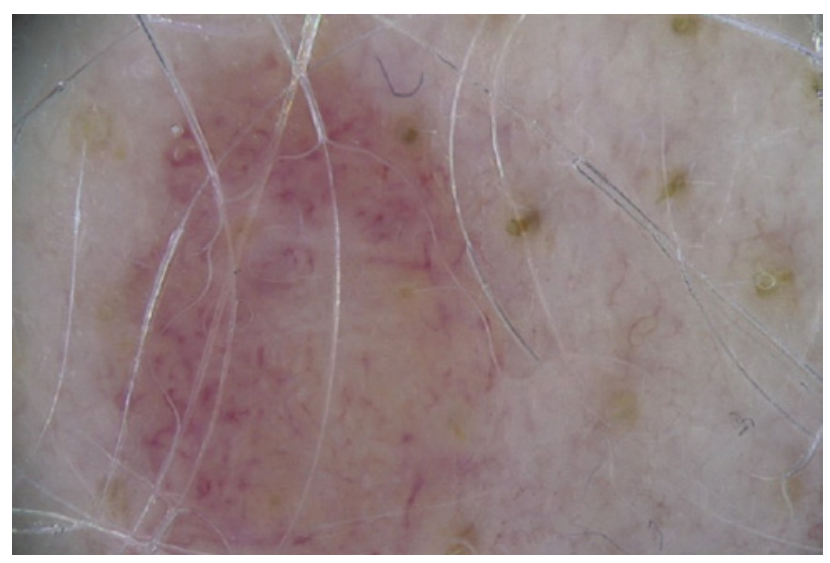

Figure 4. Nevus on back. Homogeneous light brown, yellowish pattern associated with predominantly comma-like vessels. Clinically it was a papular lesion. [Copyright: @2017 Peralta et al.]

in OCA-Ia patients. These features were also seen in our patients' nevi.

Due to reduced or absent protection from melanin, skin cancers are common in patients with albinism and the majority of these consist of squamous cell carcinoma. Although rare, melanoma has also been reported [5-8]. We want to highlight that OCA-Ia patients' nevi present a vascular pattern similar to that described for amelanotic melanoma, for which it is sometimes challenging to make a differential diagnosis-even for trained physicians.

We propose three steps to manage and follow up these kinds of nevi using dermoscopy evaluation with a comparative approach, follow-up with digital dermoscopy and reflectance confocal microscopy as complementary tools for difficult cases.

The first step is the clinical and dermoscopic evaluation using a comparative approach. It is important to evaluate all lesions in OCA-Ia patients who have multiple red Clark nevi in order to verify similar vascular patterns and colors of these nevi. If a different lesion is noticed, biopsy is mandatory. Clinical 
evaluation of these lesions can be difficult given the lack of pigmentary patterns, both under normal illumination and by dermoscopy. One way to approach patients with numerous nevi is to identify the "ugly duckling" sign (UD). When one lesion can be identified as being different from a patient's other moles, it is called the UD sign. Melanomas generally appear with a UD sign [9-10]. Another is to identify the signature nevus ( $\mathrm{SN}$ ), with signature nevi defined as the predominant group of nevi in individuals that share a similar clinical appearance [9]. The type of SN OCA-Ia patients is uniformly pink in color, seen also in those individuals with skin phototype I or II. The identification of the UD requires recognition of the $\mathrm{SN}$, these two methods actually being interrelated, and they will allow the physician to more efficiently focus diagnostic methods, such as dermoscopy and biopsy, on melanocytic lesions that are of greater concern [9].

Scope et al. [11] found that individuals tend to have one to three predominant dermoscopic nevus global patterns and defined a dominant global dermoscopic pattern, if global pattern, color and specific structures were seen in $40 \%$ or more of the patient's nevi. Patients could also have minor patterns, defined as occurring in 20 to $39 \%$ of the nevi. Later, Argenziano et al. [12] showed the importance of the dermoscopic comparative approach (to evaluate the lesions in the context of other nevi from the same patient) versus the morphologic approach (to evaluate individual lesions based only on morphologic structure) in patients with multiple nevi, concluding that the evaluation of equivocal lesions in the context of a patient's other nevi results in a lower rate of excision recommendations (a decrease from $55.1 \%$ to $14.1 \%$ ) compared with evaluation of individual lesions based on morphologic structure alone.

Therefore, we consider it crucial to perform total-body skin examinations; to observe the dermoscopic nevus global pattern(s); and to use the dermoscopic morphologic and comparative approach in order to find any suspicious lesion.

Because of the lack of pigment on nevi of OCA-Ia patients, dermoscopy may aid in diagnosis by the evaluation of vascular structures. Zalaudek et al. [13] described that comma, dotted and linear irregular vessels are suggestive of melanocytic skin tumors. Dermal nevi are typically characterized by a regular distribution of comma-like vessels. Dotted vessels are highly predictive for melanocytic skin tumors in general. Dotted vessels in red Clark nevi are loosely arranged throughout the lesion and are often associated with a few comma-like vessels, as we observed in our patients $[13,14]$.

On the other hand, it is important to consider that regularly distributed dotted vessels over a pink background associated with a reticular depigmentation is suggestive of nonpigmented Spitz nevus, but early amelanotic melanoma must be ruled out [13-15]. Additionally, the combination of dotted and linear irregular vessels over a milky red background, milky-red globules, with or without white shiny structures or reticular depigmentation is highly suggestive of invasive melanoma. Thus, excision of melanocytic tumors showing melanoma pattern is mandatory [16].

We did not observe reticular depigmentation or linear irregular vessels in our patients' lesions.

The second step is follow-up with total-body photography and digital dermoscopy. If a change is noticed, biopsy should be done. The combined use of total-body photography and digital dermoscopy called "the two-step method of digital follow-up (DFU)" in a selected population at high risk demonstrated the early detection of melanomas with a low rate of excisions. The first step is a total-body mapping, for clinical examination of the patient and total-body mapping with digital images; and the second step, a digital dermoscopy, for clinical and dermoscopic examination in real-time of all individual lesions. Digital storage of dermoscopy images of each lesion showing atypical features is recommended for later follow-up [17].

This dual modality is useful not only for the detection of melanoma with few dermoscopic criteria by DFU of dermoscopy records, but also for the detection of melanoma either presenting as new lesions or arising from nevi that were not monitored by dermoscopy. In a high-risk population, longterm follow-up is required to allow the detection of slowgrowing melanomas $[17,18]$.

Patients generally are scheduled for follow-up in 3,6 or 12 months according to the judgment of the professional who performed the evaluation. Short-term follow-up ( 3 months) is considered for individual suspicious melanocytic lesions that do not satisfy the dermoscopic criteria for the diagnosis of melanoma, while medium and long-term (6 and 12) is considered for the surveillance of patients with high or moderate risk respectively [17].

We suggest DFU for OCA-Ia patients with multiple nevi because it is the most reliable and efficient approach in detecting incipient melanoma, and DFU should be maintained in time.

The third step is to practice a RCM (reflectance confocal microscopy) as a useful complementary tool in difficult cases in order to find structures compatible with nevi or melanoma. RCM is of value as a third-step examination of clinical, dermoscopic, equivocal melanocytic lesions, because in this subset of tumors specificity is superior $[19,20]$.

Guitera et al. [19] demonstrated that diagnosis based on pure morphology is often not sufficient in the context of pink tumors and that combining dermoscopy and RCM evaluation is most likely to provide the correct diagnosis of these difficult cases. Several studies have shown that RCM may improve the accuracy in the differentiation of benign and malignant melanocytic lesions as an adjuvant technique to dermoscopy, 
and three main algorithms have been developed to apply in equivocal lesions [20].

In hypopigmented and amelanotic melanoma when dermoscopy is not able to show specific findings, RCM may render clear findings of melanoma due to the refractivity of melanoma cells, even if they are non-pigmented. Melanocytic lesions present a large number of characteristic findings visible in the upper parts of tumors, such as in the case of melanoma: pagetoid roundish or dendritic cells in superficial epidermis, atypical nests at the dermoepidermal junction, non-edged papillae and atypical nucleated cells in papillary dermis [20].

The advantage of in vivo observation of the tumor in real-time, at the bedside, is opening the clinical applications of RCM in the evaluation of melanocytic lesions, in the study of amelanotic melanomas, among others [20].

\section{Conclusion}

In conclusion, dermoscopy can be helpful to evaluate characteristics of melanocytic lesions in OCA-Ia patients, with special relevance in the vascular structures. Nevi of these patients present a vascular pattern similar to that described for amelanotic melanoma, for which it is sometimes challenging to make a differential diagnosis-even for trained physicians. Therefore, we consider crucial for the management of these patients the combination of periodic physician-based totalbody skin examinations with dermoscopy, using the comparative approach, DFU with both total-body photography and digital dermoscopy, and RCM in equivocal lesions because it has been proven to improve accuracy in early detection of melanoma in high-risk patients and reduces the number of excisions of benign lesions in these kind of patients.

\section{References}

1. Grønskov K, Ek J, Brondum-Nielsen K. Oculocutaneous albinism. Orphanet J Rare Dis. 2007;2:43.

2. Caldarola G, Fania L, Fossati B, et al. Dermoscopy of melanocytic lesions in patients affected by oculocutaneous albinism: a case series. Dermatology. 2013;226:358-361.

3. Bakos RM, Argenziano G, Zalaudek I, et al. Dermatoscopy of pigmented melanocytic nevi in patients with oculocutaneous albinism. J Am Acad Dermatol. 2009;60:487-489.
4. Ortonne JP. Trastornos pigmentarios. In: Bolognia JL, Jorizzo JL, Rapini RP. Dermatology. 1st ed. Spain: Elsevier; 2004:955-956.

5. Perry PK, Silverberg NB. Cutaneous malignancy in albinism. Cutis. 2001;67:427-430.

6. Streutker CJ, McCready D, Jimbow K, From L. Malignant melanoma in a patient with oculocutaneous albinism. J Cutan Med Surg. 2000;4:149-152.

7. Wu CY, Gao HW, Chiang CP. Malignant amelanotic melanoma developing from an intradermal naevus in a patient with oculocutaneous albinism. Clin Exp Dermatol. 2009;34:590-593.

8. Zalaudek I, Argenziano G, Mordente I, et al. Nevus type in dermoscopy is related to skin type in white persons. Arch Dermatol. 2007;143: 351-356.

9. Suh KY, Jean L, Bolognia JL. Signature nevi. J Am Acad Dermatol. 2009;60:508-514.

10. Scope A, Dusza SW, Halpern AC, et al. The "ugly duckling" sign: agreement between observers. Arch Dermatol. 2008;144:58-64.

11. Scope A, Burroni M, Agero AL, et al. Predominant dermoscopic patterns observed among nevi. J Cutan Med Surg 2006;10:170-4.

12. Argenziano G, Catricalà $C$, Ardigo $M$, et al. Dermoscopy of patients with multiple nevi: Improved management recommendations using a comparative diagnostic approach. Arch Dermatol. 2011;147:46-49.

13. Zalaudek I, Kreusch J, Giacomel J, et al. How to diagnose nonpigmented skin tumors: a review of vascular structures seen with dermoscopy: part I. Melanocytic skin tumors. J Am Acad Dermatol. 2010;63:361-374.

14. Menzies SW, Kreusch J, Byth K, et al. Dermoscopic evaluation of amelanotic and hypomelanotic melanoma. Arch Dermatol. 2008;144:1120-1127.

15. Carrera C, Palou J, Malvehy J, et al. Early stages of melanoma on the limbs of high-risk patients: clinical, dermoscopic, reflectance confocal microscopy and histopathological characterization for improved recognition. Acta Derm Venereol. 2011;91:137-146.

16. Zalaudek I, Kittler H, Hofmann-Wellenhof R, et al. "White” network in Spitz nevi and early melanomas lacking significant pigmentation. J Am Acad Dermatol. 2013;69:56-60.

17. Salerni G, Carrera C, Lovatto L, et al. Benefits of total body photography and digital dermatoscopy ("two-step method of digital follow-up") in the early diagnosis of melanoma in patients at high risk for melanoma. J Am Acad Dermatol. 2012;67:17-27.

18. Puig S, Malvehy J. Monitoring patients with multiple nevi. Dermatol Clin. 2013;31:565-577.

19. Guitera P, Menzies SW, Argenziano G, et al. Dermoscopy and in vivo confocal microscopy are complimentary techniques for the diagnosis of difficult amelanotic and light colored skin lesions. $\mathrm{Br}$ J Dermatol. 2016;175(6):1311-1319.

20. Carrera C, Puig S, Malvehy J. In vivo confocal reflectance microscopy in melanoma. Dermatol Ther. 2012;25:410-422. 